Under review. Philosophical Transactions of the Royal Society B, theme issue on "Foundations of Cultural Evolution" edited by Eva Boon, Piet van den Berg, Lucas Molleman \& Franjo Weissing. 27 August 2020

\title{
The Cultural Evolution of Cultural Evolution
}

\author{
Jonathan Birch $^{1}$ and Cecilia Heyes ${ }^{2}$ \\ ${ }^{1}$ Department of Philosophy, Logic and Scientific Method \\ London School of Economics and Political Science \\ Houghton Street, London WC2A 2AE, UK \\ j.birch2@1se.ac.uk \\ ${ }^{2}$ All Souls College and Department of Experimental Psychology \\ University of Oxford \\ Oxford OX1 4AL, UK \\ cecilia.heyes@,all-souls.ox.ac.uk
}

Keywords: Cognitive gadgets; cultural evolution; evolution of cognition; learning bias; social learning strategy; metacognition 
Abstract: What makes fast, cumulative cultural evolution work? Where did it come from? Why is it the sole preserve of humans? We set out a self-assembly hypothesis: cultural evolution evolved culturally. We present an evolutionary account that shows this hypothesis to be coherent, plausible, and worthy of further investigation. It has the following steps: (0) in common with other animals, early hominins had significant capacity for social learning; (1) knowledge and skills learned by offspring from their parents began to spread because bearers had more offspring, a process we call CS1 (or Cultural Selection 1); (2) CS1 shaped attentional learning biases; (3) these attentional biases were augmented by explicit learning biases (judgements about what should be copied from whom). Explicit learning biases enabled (4) the high-fidelity, exclusive copying required for fast cultural accumulation of knowledge and skills by a process we call CS2 (or Cultural Selection 2), and (5) the emergence of cognitive processes such as imitation, mindreading and metacognition 'cognitive gadgets' specialised for cultural learning. This self-assembly hypothesis is consistent with archaeological evidence that the stone tools used by early hominins were not dependent on fast, cumulative cultural evolution, and suggests new priorities for research on 'animal culture'. 
How did human cultural evolution get off the ground? It would be an exaggeration to say there is nothing at all like it elsewhere in the natural world. Cetaceans[1-4], great apes[5, 6] and other animals[7-11] also pass skills down the generations through social learning, leading to stable differences between sub-populations. These differences are often called "traditions." $[2,10]$ Young bottlenose dolphins learn foraging techniques from their parents[12], young chimpanzees learn from older group members how to extract ants and termites from their mounds with sticks[13]. But human cultural evolution (see Table 1) is different. It is cumulative: small improvements to skills and technologies accumulate, resulting in products of such complexity that no one individual could possibly have designed them alone, without any learning from others[14-17]. Examples include canoes, spears, fire control, detoxification of bitter manioc, and, more recently, steam engines, computers, and satellites. Moreover, the accumulation is fast: improvements spread rapidly through populations, no longer tied to the timescale of biological generations. What makes fast, cumulative cultural evolution work? Where did it come from? And why is it the sole preserve of humans?

There is an emerging consensus that fast cultural accumulation draws on a suite of cognitive mechanisms including selective social learning, imitation, language, mindreading (or theory-of-mind) for teaching, metacognition and normative cognition, and that humans have evolved uniquely sophisticated versions of these mechanisms[18, 19]. But what explains the origin of these mechanisms?

A popular, influential type of answer appeals to gene-culture co-evolution. This occurs when the genetic composition of a population responds to changes in the cultural environment, leading to yet further changes in the cultural environment, and so on. The idea is often illustrated by the case of lactose tolerance: genes for lactose tolerance followed the spread of dairy farming, and enabled yet more dairy farming. Boyd and Richerson [20] proposed that some of the cognitive mechanisms involved in human cultural evolutionincluding the mechanisms mediating conformist bias - evolved, like lactose tolerance, by gene-culture co-evolution. This has remained a central tenet of the "California school" of cultural evolution that Boyd, Richerson, Henrich and their collaborators have built over the past thirty years.

While gene-culture co-evolution has received a great deal of attention, much less has been given to its purely cultural counterpart. We call this (borrowing a term from Muthukrishna and Henrich [21]) culture-culture co-evolution. As we define it here, cultureculture co-evolution occurs when a culturally inherited cognitive mechanism evolves in 
response to a cultural environment, altering that environment and enabling further evolution of the cognitive mechanism, without any underlying genetic change.

Our aim here is to explain the basic idea of culture-culture co-evolution and to argue that it is worthy of sustained empirical investigation. Indeed, when it comes to explaining the origins of cumulative culture, we think it may even provide a better explanation than geneculture co-evolution.

\section{Cultural selection, fast and slow}

At the core of our hypothesis about the origins of cumulative culture is a distinction between two types of cultural selection. A Darwinian process is one that relies on blind variation and selective retention (Supplement 1). In genetic evolution, "selective retention" always involves the differential survival and/or reproduction of individuals. But in cultural evolution, there are two different types of "selective retention" operating at different timescales.

The first, slower type is natural selection on culturally inherited variation [16, 22]. We will call this Cultural Selection 1 or CS1. CS1 is closely analogous to natural selection on genetic variation. When offspring spend a long time learning practical skills and ecological knowledge from their parents, valuable skills and knowledge will tend to spread through the population for the simple reason that their bearers will tend to have more offspring. Just as in a traditional process of natural selection, change is driven by differences in the number of biological offspring an organism produces. However, unlike in a traditional process of natural selection, the differences in reproductive output are caused by inherited differences in cultural variants, not genes, and they are transmitted from parents to offspring through social learning rather than genetic inheritance.

The second, faster type of Darwinian process occurs when individuals compete with each other for learners, so that individuals who recruit more learners can be said to have higher cultural fitness [23]. We will call this Cultural Selection 2, or CS2. Roughly, an agent's cultural fitness, with respect to a specific cultural trait, is the number of learners to whom it transmits its variant of that trait through social learning [22-25]. When learners are choosing from a wide range of potential models, and the models are competing for learners, there is potential for fast accumulation of small improvements, because the process need not be tied to the timescale of biological reproduction. We think CS2 lies at the heart of "cumulative culture" as we know it today. Small improvements to existing techniques spread not because they necessarily increase anyone's reproductive success, but because they allow models to attract more learners. 
Although we have set out the CS1/CS2 at the individual level, the same distinction can be drawn at the group level. In other words, the CS1/CS2 distinction cross-cuts the distinction between individual selection and group selection. In the group-level version of $\mathrm{CS} 1$, change is driven by biological fitness differences between groups, and these differences are due to culturally inherited variation in group-wide patterns of behaviour. In the grouplevel version of CS2, change is driven by cultural fitness differences between groupsdifferences in their ability to attract new migrants who will learn their ways. This group-level version of CS2 will be important later on.

The CS1/CS2 distinction allows us to pose our question like this: at some point in human evolution, CS1 began to be supplemented by CS2, allowing fast cultural accumulation. Was this transition driven by gene-culture co-evolution, or did CS1 itself assemble the mechanisms that made CS2 possible? If the latter is the case, then the mechanisms that enable cumulative culture were products of culture-culture co-evolution. Cumulative culture was, in this specific sense, "self-assembling".

\section{A self-assembly hypothesis}

According to our self-assembly hypothesis, the role of genetic evolution, though important, was limited. Genetic evolution driven by increasing climatic variability and environmental change gave us larger brains[26], longer childhoods[27], and more powerful domain-general cognitive resources. Associative learning, working memory and inhibitory control were all dialled up, leading, eventually, to a distinctive capacity for slow, deliberative, explicit cognition that made heavy use of working memory [28-31]. These genetically-based upgrades to domain-general cognitive resources enabled early hominins to learn more efficiently the information they needed to hunt and gather in a changing and variable environment. But, on our hypothesis, these genetic changes were not responsible for shaping specialized cognitive mechanisms for accelerating cultural evolution. Cognitive mechanisms like imitation, mindreading and normative cognition emerged later via cultural evolutionary processes. Relatively simple cognition got fast cultural evolution off the ground. Learners were initially driven by attentional processes to copy better models and small improvements in technique; they did not make explicit comparisons between models or consciously recognize improvements. They had "competence without comprehension" [32].

Our hypothesis emphasizes the importance of culture-culture co-evolution. We do not assume that specialized cognitive mechanisms such as imitation have been genetically assimilated to any substantial degree. This is because we think the present-day evidence from 
developmental psychology and cognitive neuroscience points towards these mechanisms being culturally inherited now (Supplement 3, [33]). To the extent that this is plausible, it is important to have viable evolutionary hypotheses that do not rely on genetic assimilation.

Our hypothesis has a basic platform — a starting point shared with other great apesand five steps from that basic platform to fast, cumulative culture (Figure 1).

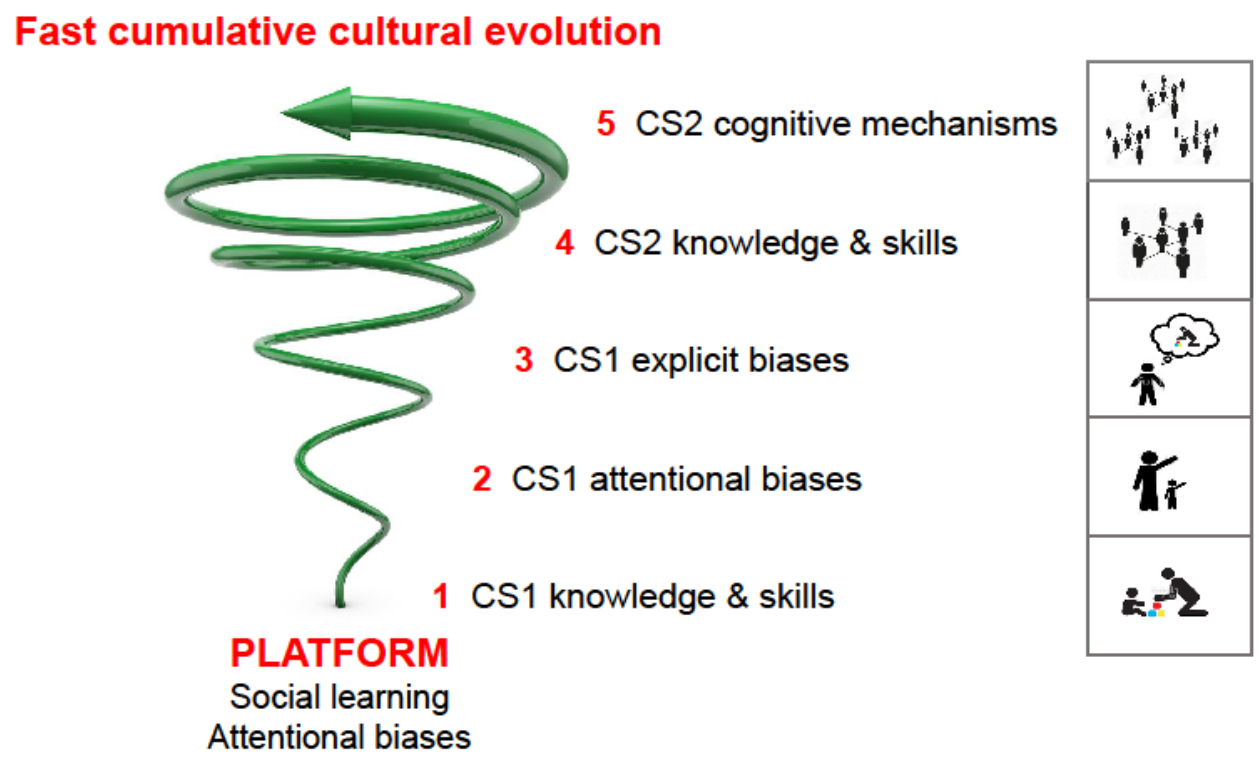

Figure 1. The self-assembly hypothesis. Culture-culture coevolution produces fast cumulative culture in five steps. Cultural Selection 1 (CS1) initiates the co-evolutionary process. First knowledge and skills (Step 1), then attentional social learning biases (Step 2), then explicit social learning biases (Step 3), are socially inherited from biological parents and spread through the population because their bearers tend to have more offspring.

Subsequently, Cultural Selection 2 (CS2) also contributes. Enhanced knowledge and skills (Step 4) and cognitive mechanisms (Step 5) are socially inherited from unrelated individuals and spread through the population because some models are more successful than others in competition for learners. The five-step process not only produces fast cumulative culture, but is itself cumulative: each step augments, rather than replaces, the previous step (green arrow). The schematic figures on the right represent typical social interactions in each step. See text for details.

\section{Step 0: The basic platform}

The human lineage, at the time of its divergence from the other great apes about six million years ago, would have possessed a significant capacity for social learning. Like a wide range of animal species alive today, our earliest human ancestors could learn by observing the behaviour of other agents that some places and objects are worth exploring (stimulus enhancement $[34,35]$ ), while others are either dangerous or rewarding (observational 
conditioning[36, 37]). They could also learn socially what the outcomes of actions are likely to be (observational learning[38, 39]): prodding a hive releases bees, and digging in certain areas reveals tubers [40, 41].

These social learning capacities were once thought to depend on specialised cognitive processes, but recent evidence indicates that they are based on the same associative mechanisms as those involved in asocial learning [38, 42-47]. These mechanisms are powerful but, in the form present among our earliest ancestors, they do not allow high fidelity copying of fine bodily movement and technique.

Early hominins would also have possessed structural and attentional social learning biases. A structural bias is a tendency to learn from some models rather than others due to social structure and geography [48-50]. Juveniles would have learned most from their mothers, and to a lesser extent from other members of their immediate residential group, simply because they spent more time in close proximity to these agents than to others. An attentional bias is a tendency to learn from some models rather than others because they are particularly attention-grabbing [30,51]. These attention-grabbing actors or actions might be intrinsically salient (large, noisy, pungent)[52], close to salient rewards (e.g. close to food $[53,54])$ or previously associated with reward (e.g. their actions tend to produce or release food[55]).

Structural and attentional biases, like the social learning they modulate, are based on evolutionarily ancient, domain-general cognitive mechanisms [56, 57]. The social learning biases found in fish, birds and small mammals $[48,58]$ can be explained by attentional processes that bias social learning in the same way as asocial learning, and that evolved long before hominins appeared on the scene [57]. Nonetheless, we should not underestimate the power of attentional biases. They can produce patterns which, at the population level, fit the description of what cultural evolution theorists call conformist bias, prestige bias and payoff bias $[16,59,60]$. For example, suppose a child learns to associate a particular adult's behaviour with subsequent food rewards and, as a result, attends preferentially to that adult's behaviour. They will end up displaying a simple form of payoff-biased learning without needing to make any explicit judgements about whose behaviour leads to payoffs [55].

\section{Step 1: Cultural Selection 1 of knowledge and skills}

A capacity for social learning, and for simple learning biases, can be found in a wide range of species, and is clearly not sufficient for fast, cumulative culture. What made hominins different? 
For early hominins, learned knowledge and skills were crucial for survival, and, with the extension of childhood, juveniles had more opportunity to acquire them vertically, primarily from their mothers [61]. Consequently, socially learned knowledge and skills began to spread through populations for the simple reason that their bearers tended to have greater reproductive fitness. Individuals who learned, for example, a more effective foraging technique from their mothers had better nutrition and more viable offspring, and passed the technique on to each of those descendants. CS1 of knowledge and skills became increasingly powerful and important.

This does not mean that CS1 is uniquely human. In fact, CS1 appears to be occurring now among bottlenose dolphins. Some wild dolphins in Shark Bay, Western Australia, use marine sponges, worn on their closed rostrum (beak), to probe the sea floor for food[62]. There is evidence that this foraging technique is transmitted vertically through social learning from mothers to their female offspring[12], and that mothers who forage in this way have more offspring than mothers who do not [63]. If it is confirmed that the technique is spreading by selection on cultural variation, these dolphins could be an excellent model system for studying the very earliest stages of hominin cultural evolution.

\section{Step 2: Cultural Selection 1 of attentional learning biases}

CS1 can be cumulative, but the accumulation will be slow (Supplement 2). Since it relies on differential biological reproduction, CS1 occurs on broadly the same timescale as genetic evolution (although the supply of variation may differ: new cultural innovations may appear more or less frequently than genetic mutations). What it provides is a process that can drive the evolution of new cognitive mechanisms that accelerate cultural evolution. In the first accelerating innovation, learned attentional biases - cognitive rather than physical skillsbecame subject to CS1.

CS1 acting on knowledge and physical skills (Step 1) produced an increasing number of valuable, socially-learnable traits in human populations: knowledge and skills that it would be advantageous for juveniles to learn from models other than one's own parents. Ancient attentional biases (Step 0) were available to guide juveniles when they were learning from unrelated adults and peers (oblique transmission and horizontal transmission), but their choice of models became yet more adaptive when they began to socially learn attentional biases from their parents. For example, they no longer had to learn for themselves, by trialand-error, that it is beneficial to attend more to females for foraging skills and males for hunting skills (if indeed it was), or to go to elders rather than peers for rare ecological 
information. Instead, they could learn these biases by tracking the social attention of their parents in different task contexts.

There is evidence that contemporary humans can socially learn attentional biases. Specifically, we can learn to attend more to some agents than others by following the gaze of third parties $[64,65]$. For example, when given choices of foods to consume and objects to manipulate, 3- and 4-year-old children are more likely to learn from an adult to whom they have seen other adults attending than from an adult who was ignored [64]. Nonhuman primates have attentional biases that influence their choice of models $[66,67]$ but, as far as we are aware, no one has asked whether nonhuman animals can socially learn attentional biases. For example, there is evidence that vervet monkeys attend more to female than male models when learning how to open a box to obtain food[66] but the origin of this attentional bias is not known. Does each vervet learn through its own efforts that the behaviour of females is more likely than the behaviour of males to have a desirable outcome, or can young vervets learn to attend more closely to females by watching the watching behaviour of adults?

The existing evidence leaves a great deal of uncertainty as to when the ability to socially learn attentional biases first evolved. We conjecture that it was in place in Homo erectus well before 700,000 years ago, when the next steps in our account begin. It may have evolved much earlier. Although we suspect that socially learned attentional biases have been far more important for hominins than for other species, we do not rule out the possibility that some other animals do learn attentional biases from their parents, and that these learned attentional biases have been shaped by CS1.

Research on "shared attention" and "joint attention" in contemporary children often assumes that these phenomena require understanding of others' psychological states [68]. In contrast, step 2 in our self-assembly model does not require mindreading. For social learning of attentional bias, learners need to track models looking behaviour, not their mental states.

In so far as attentional biases are transmitted from parents to offspring, they can be targets of CS1. Parents with valuable attentional biases will have greater reproductive success, and the biases will spread.

\section{Step 3: Cultural Selection 1 of explicit learning biases}

We come now to the steps that we propose to be unique to the hominin lineage. As noted above, we agree with the received wisdom that human cognitive evolution involved genetically-based upgrades to domain-general cognitive resources, leading to a capacity for slow, deliberative, explicit cognition that made heavy use of working memory. The basic 
logic of our self-assembly hypothesis does not depend on any specific assumptions regarding when this happened. We suspect, however, that Late Acheulean lithic technology is a sign that a form of explicit cognition was in place by around 700,000 years ago, the time of Homo heidelbergensis. Neuroimaging studies suggest that, in modern humans, success in Late Acheulean toolmaking techniques relies on working memory [69-72]. We cannot be sure that H. heidelbergensis would have learned and executed Acheulean toolmaking skills in the same way we do, but it is a reasonable conjecture.

Once explicit cognition is present, it enables a new type of learning bias on which CS1 can get to work - explicit learning biases. Explicit learning biases are internally represented rules of thumb. They may include highly specific rules such as: "find individual $X, Y$ or $Z$ to observe the manufacture of cleavers, and individual $A, B$ or $C$ to observe the manufacture of handaxes." Some rules may refer learners to specific models (“ $X, Y$ or $Z$ ”), while others may refer learners to a general type of model (such as "learn flint knapping from the person who makes the most symmetric shapes"). Explicit learning biases require a capacity for conceptual representation (e.g. possession of concepts referring to specific types of person, tool, and shapes), but it is not clear that they require language. Language could have come along later.

Attentional biases, of the kind that were important in Steps 1 and 2, are limited in their specificity and accuracy. They lead learners to better than average models from the pool of nearby options, but they will struggle to lead learners to the best models in a sizeable social group. By relating cranial volume to social network size, Gamble, Gowlett and Dunbar estimate that $\mathrm{H}$. heidelbergensis lived in social networks of 100-150 recognized individuals $[73,74]$. A more recent estimate, incorporating archaeological data alongside cranial volume, revised this downwards to 60-120 [75]. But even a group size of 60 is enough to create a problem. As group size grows, it becomes increasingly difficult for a juvenile to learn who the best experts are at specific skills purely by tracking the gaze of their parents. Juveniles might learn to attend to elders when uncertain about an important ecological fact, but they will not reliably find their way to the most knowledgeable elder. The problem is that they are not making explicit judgements about who should, and who should not, be copied in particular domains. It is simply that their attention is reliably drawn to some models rather than others by relatively low-level, domain-general psychological processes. Because learners are not reliably able to find the most technically accomplished models in large groups, in Steps 1 and 2 we do not yet have conditions in which small improvements to an established technique can spread reliably and rapidly. 
In Step 3, explicit learning biases allow juveniles to reliably access the best models available, and to dedicate their learning efforts to those specific models. A learner who can reliably find the most technically accomplished models will gain biological fitness benefits from doing so, as long as their superior technique can be learned. This provides a selection pressure in favour of explicit learning biases, since they can overcome the limitations of attentional learning biases.

Although genetic changes of some kind will have been involved in the origin of explicit cognition, there is no reason to think that any further genetic changes are required for explicit learning biases, once explicit cognition in general exists. Explicit cognition brings with it a versatile capacity to represent rules. Rules about learning (such as copy the majority) can be learned, just like straightforwardly technical rules (such as strike flint against pyrite to make a spark). If the learning rules are passed down the generations from parents to offspring, CS1 will be able to shape them, gradually making them increasingly adaptive. We envisage this refinement of explicit learning biases through CS1 as a gradual process taking place over hundreds of thousands of years, beginning around 700,000 years ago.

There is evidence that explicit social learning biases - judgements about who to copy - are crucial to fast cultural accumulation $[51,76,77]$. They are used, in addition to attentional learning biases, by adult humans alive today[78-80], but as yet there is no evidence that they are used by children younger than 4 or 5-years-old[81] or by nonhuman animals.[57]

\section{Step 4: Cultural Selection 2 of knowledge and skills}

With accurate, specific, explicit learning biases, assembled by CS1, comes the possibility of fast, cumulative cultural evolution of knowledge and skills. An explicit rule can be highly task-specific: it can pertain to the manufacture of a particular tool, for example, and may identify a correlate of success that is very specific to that task, such as the symmetry of the end-product. This task-specificity means high quality models can be reliably identified, leading to greater exclusivity. A learner equipped with a model selection strategy that reliably picks out high quality models for particular task-specific skills has more to gain from investing effort in copying the specific technique of a specific individual, rather than hedging their bets and learning from as many different models as possible [51, 82].

High fidelity copying of small improvements is the type of copying that allows fast accumulation. Because learners are very discriminating - they choose only the best models in the relevant task domain, and learn exclusively or nearly exclusively from them-small 
improvements in technique can spread reliably and rapidly without being explicitly recognised by the learner as improvements. They spread because they make a substantial difference to the cultural fitness of their bearers: their success in attracting learners who are selecting their models using explicit learning biases. If a small improvement to an existing technique promotes the correlate of success tracked by the learning bias (e.g. the symmetry of the end-product), learners will gravitate towards the innovator. Small improvements to existing techniques will be retained in the population and will spread widely, creating many new sites for new small improvements, which will in turn be retained and copied.

This is a process with a Darwinian character, but one in which change is driven not by differences in reproductive success but by differences between models in the number of learners they attract. This is Cultural Selection 2 (CS2): cultural change driven by culturally inherited differences in cultural fitness. CS2 is the engine of fast, cumulative cultural change. We do not think there was a single, special moment when CS2 "took off", and we do not think that explicit learning biases were the only crucial ingredient. Life history mattered: as juvenile development lengthened, juveniles were able to invest more and more time in learning knowledge and skills, increasing the opportunities for CS2. Demography also mattered: in large, richly interconnected populations, there were more heads in which innovation could occur, more heads in which small improvements could be retained, and more to gain from seeking out the best models for particular skills [83]. We envisage CS2 becoming gradually more important over time, as explicit learning biases become increasingly task-specific and exclusive, as the juvenile period of human life history becomes increasingly long, and as populations become larger and more richly interconnected. Levallois prepared core techniques may be an archaeological signature of cumulative CS2 [19].

\section{Step 5: Cultural Selection 2 of cognitive mechanisms}

CS2 is not limited to selection of small improvements to knowledge and skills. Small improvements to cognitive mechanisms that accelerate cultural accumulation, including imitation, mindreading and metacognition, can also increase in frequency.

How might this work? The idea is that the cognitive mechanisms of cultural learning are themselves learned skills, or "cognitive gadgets" (Supplement 3[30]), and learners are selective in who they learn these skills from. Let us focus on the case of imitation-copying the topography of body movements. Imitation involves learning associations between how movements feel from the inside and how they look from the outside, solving the 
correspondence problem. An adult can help a child to develop imitation in various ways: by copying their own movements (i.e. by imitating them) in their sight, by giving them opportunities to practice copying bodily movements with appropriate feedback, and by seeding the child's environment with reflective surfaces that allow the child to see what they are doing [84].

In contrast to some other skills, a child will not learn imitation from a single model. More plausibly, the child will learn it gradually from a wide range of adults and peers in their residential group. Because of this, we think a process of model selection operating at the group level may have been particularly important. Provided migration between residential groups is possible (and, in contemporary hunter-gatherers, it is common) the migrating parent can choose the group in which they live, and can therefore choose the child's set of models. The parent will choose using learned explicit rules, such as move where people look healthiest, which they can put into practice at large seasonal aggregations of many residential groups [85]. The parent's choice will be influenced by, among other factors, the imitative abilities of the group, in so far as groups with better imitative abilities will be more cooperative and have better ecological knowledge and skills. Groups with better imitative abilities will attract more migrants, and therefore more learners. This is a form of CS2, since it relies on model selection, but the model selection involves evaluations of whole groups rather than individuals. Moreover, since imitation is transmitted in part through models imitating learners, we predict that models who are better imitators will also be better at transmitting their superior imitative abilities to those who spend time around them-a positive feedback loop.

In short, groups whose members have better cognitive mechanisms for high-fidelity, selective copying will attract more migrants and grow in size at the expense of others. As groups get larger, there are more and more heads in which a new, small innovation might occur. This has the potential to further accelerate the CS2 of knowledge and skills at the level of individuals within those groups, making the group as a whole yet more attractive to new migrants - another positive feedback loop.

\section{Conclusion}

The self-assembly hypothesis offers three new answers to the question: Why is cumulative culture uniquely human? They are not mutually exclusive, and each answer is empirically testable. 
First, it is possible that CS1 of knowledge and skills (Step 1) has rarely evolved because few species have had a social structure that gives juveniles the opportunity to learn a lot from their parents with high fidelity $[48,86]$. This would be supported by evidenceperhaps modelled on recent work with dolphins[2, 4, 12, 63] — showing that CS1 of knowledge and skills is more common in species where juveniles spend most of their time with biological relatives of the previous generation.

Second, it may be difficult to transition from CS1 of knowledge and skills to CS1 of attentional biases (Step 2) because variants required to track parental attention are rarely available. In humans, attention tracking is enhanced by inborn tendencies to orient to faces [87] and voices[88] and possibly by reduced scleral colouration [89]. A priority for future research is to find out which extant species are capable of socially learning attentionally biases (for example, to learn copy females by observing the orienting behaviour of third parties[66]), and whether, in human and nonhuman populations, such biases can be vertically inherited in a way that enhances biological fitness.

Third, there may be a roadblock at the emergence of explicit social learning biases (Step 3). These require explicit cognition of a kind that may or may not be dependent on language [51]. To find out, we need research with nonhuman animals that does not merely document social learning biases, but is designed to distinguish biases mediated by implicit (attentional) from explicit cognition.[57]

The self-assembly hypothesis suggests that fast, cumulative culture emerged slowly by means of CS1. In this respect, it is consistent with archaeological evidence that the stone tools used by early hominins (Oldowan and Acheulean) were not dependent on fast, cumulative culture $[70,90,91]$.

The search for animal culture[9, 11] has been dominated by the idea that patterns of geographical variation in socially learned behaviour, and in particular the diffusion of qualitative novelty, are the most important things to investigate $[3,7,8,46]$. There has been interesting developmental work on opportunities for social learning in free-living primates, e.g. $[92,93]$, but relatively little attention has been given to vertical cultural inheritance: How much do offspring learn from their parents? Are small improvements retained or not? The self-assembly hypothesis suggests that these questions matter, because the roots of fast cumulative culture are more likely to be found in species that, due to their social structure, have significant potential for high-fidelity vertical cultural inheritance under free-living conditions. This would be evident in family-specific behaviours: techniques passed down the 
generations of a family group and varying between families, with small improvements being retained.

We have argued that the self-assembly hypothesis is coherent, plausible, and empirically testable. It contrasts with a standard gene-culture co-evolution story by giving a central role to culture-culture co-evolution, with a fairly circumscribed role for genetic change. This type of account is favoured by evidence that cultural learning is culturally inherited today (Supplement 3), and can be tested further through empirical research on the behaviour of human and nonhuman animals.

Let us now return to the questions we started with. What makes fast, cumulative cultural evolution work? Our answer is CS2: models varying in the number of learners they attract, with small, unobvious improvements being copied faithfully and more often, allowing those improvements to spread rapidly through populations. Where did it come from? We suggest that CS2 came from CS1: a simpler, slower process in which knowledge and skills, then attentional biases, then explicit biases were inherited by juveniles predominantly from their biological parents. Why is cumulative cultural evolution the sole preserve of humans? Because there are several points along the path from a platform of basic social learning capacities to CS2 where the conditions have to be just right. Other species, throughout the animal kingdom, may be at various points on the path, but only one has made it to the end.

Acknowledgements

We are grateful to Christine Caldwell, Russell Gray, Kevin Laland, Michael Muthukrishna, Kim Sterelny, Claudio Tennie, Tobias Uller and two anonymous reviewers for their comments and advice. JB thanks an audience at the "Evolution Evolving" conference (University of Cambridge, April 2019) for their questions and feedback. JB's research was funded by a Philip Leverhulme Prize from the Leverhulme Trust. 
Table 1. Glossary

\begin{tabular}{|c|c|}
\hline Attentional bias & A social learning bias mediated by domain-general attentional processes \\
\hline Asocial learning & $\begin{array}{l}\text { Learning without the assistance of other agents. Also known as "individual } \\
\text { learning" }\end{array}$ \\
\hline Cognitive gadget & A domain-specific cognitive process shaped by cultural evolution \\
\hline Conformist bias & $\begin{array}{l}\text { A social learning bias in favour of variants that are currently prevalent among } \\
\text { available models }\end{array}$ \\
\hline Cultural evolution & Change in the frequencies of cultural variants over time \\
\hline Cultural fitness & $\begin{array}{l}\text { The number of learners an individual model succeeds in attracting, weighted } \\
\text { by the model's degree of influence over the learner }\end{array}$ \\
\hline Cultural inheritance & $\begin{array}{l}\text { The transmission down the generations of cultural variants, leading to } \\
\text { persisting cultural differences between populations }\end{array}$ \\
\hline Cultural learning & $\begin{array}{l}\text { Social learning mediated by cognitive mechanisms that are specialized for } \\
\text { promoting cultural inheritance }\end{array}$ \\
\hline Cultural Selection 1 (CS1) & $\begin{array}{l}\text { A Darwinian process in which cultural variants spread because they cause } \\
\text { their bearers to have more biological offspring, and because offspring learn } \\
\text { from their parents }\end{array}$ \\
\hline Cultural Selection 2 (CS2) & $\begin{array}{l}\text { A Darwinian process in which cultural variants spread because they cause } \\
\text { their bearers to attract more learners, giving them higher cultural fitness }\end{array}$ \\
\hline Cultural variant & $\begin{array}{l}\text { Anything that can be learned socially (e.g. psychological processes, artefacts, } \\
\text { skills, habits, customs, rituals, ideas, beliefs, values) }\end{array}$ \\
\hline Cumulative cultural evolution & $\begin{array}{l}\text { Cultural evolution in which small improvements to existing cultural variants } \\
\text { spread through populations, gradually leading to complex adaptive products } \\
\text { that no single individual could have designed from scratch }\end{array}$ \\
\hline $\begin{array}{l}\text { Domain-general cognitive } \\
\text { mechanism }\end{array}$ & $\begin{array}{l}\text { A cognitive mechanism that works in the same way across a broad range of } \\
\text { tasks and contexts, e.g. social and non-social }\end{array}$ \\
\hline $\begin{array}{l}\text { Domain-specific cognitive } \\
\text { mechanism }\end{array}$ & $\begin{array}{l}\text { A cognitive mechanism that works in one task or context and less efficiently } \\
\text { or not at all in others, e.g. social or non-social }\end{array}$ \\
\hline Explicit bias & $\begin{array}{l}\text { A social learning bias mediated by a domain-specific psychological rule such } \\
\text { as copy the majority }\end{array}$ \\
\hline Genetic assimilation & $\begin{array}{l}\text { A process whereby environmentally induced phenotypic variation acquires a } \\
\text { genetic basis }\end{array}$ \\
\hline Genetic evolution & Change in the frequencies of genes over time \\
\hline High-fidelity transmission & $\begin{array}{l}\text { The learning of a cultural variant with sufficiently few errors that even small, } \\
\text { unobvious improvements will be retained }\end{array}$ \\
\hline Horizontal transmission & $\begin{array}{l}\text { The learning of a cultural variant by a member of one generation from } \\
\text { another member of the same generation }\end{array}$ \\
\hline Oblique transmission & $\begin{array}{l}\text { The learning of a cultural variant by a member of a younger generation from } \\
\text { a member of an older generation who is not a biological parent }\end{array}$ \\
\hline Payoff bias & $\begin{array}{l}\text { A social learning bias in favour of learning cultural variants that effectively } \\
\text { yield rewards }\end{array}$ \\
\hline Prestige bias & $\begin{array}{l}\text { A social learning bias in favour of learning from models who are already } \\
\text { successful in attracting learners and have markers of that success }\end{array}$ \\
\hline Social learning & Learning assisted by observation of, or interaction with, other agents \\
\hline Social learning bias & $\begin{array}{l}\text { Any mechanism that biases an individual's social learning away from one } \\
\text { cultural variant and towards another. Also known as "social learning } \\
\text { strategy", "learning bias", and "transmission bias" }\end{array}$ \\
\hline Vertical transmission & The learning of a cultural variant from a parent by its biological offspring \\
\hline
\end{tabular}




\section{Supplement 1 - Cultural evolution and population thinking}

Cultural evolution research is currently in a period of rapid development, and various different schools of thought co-exist in the field. ${ }^{1-8}$

Most cultural evolutionists agree on the importance of "population thinking": cultural change can be understood by looking at the dynamics of social learning processes acting on populations. But many population thinkers downplay the importance of Darwinian selection processes. ${ }^{9,10}$ For example, Sperber and colleagues ${ }^{11,12}$ have developed a non-Darwinian framework based on the concept of a "cultural attractor". These non-Darwinian approaches are not our focus here.

Defenders of Darwinian (or "selectionist") approaches, pioneered by Donald Campbell ${ }^{13,14}$ in the 1960 s and 1970s, hold that Darwinian processes have a special role. They argue that, just as in the case of genetic evolution, there are many causes of cultural change, but only a Darwinian process of "blind variation and selective retention"13 is able to generate complex, well-adapted cultural products without the need for intelligent design. ${ }^{15,16,17,18}$

Darwinian approaches can themselves be divided into meme-centred and individualcentred approaches. ${ }^{19,20}$ The meme-centred approach regards the cultural inheritance as particulate, like genetic inheritance, and models the flow of these particles through populations. ${ }^{6,21}$ The individual-centred approach does not assume that cultural inheritance is particulate. It focusses on learners and the people from whom they learn ("models"), looking in a more holistic way at the inheritance channel between a model and a learner. Sometimes models that succeed in attracting larger numbers of learners are said to have higher cultural fitness (Supplement 2).

References

1 Henrich, J. The Secret of Our Success: How Culture is Driving Human Evolution, Domesticating Our Species, and Making Us Smarter. (Princeton University Press, 2016).

2 Laland, K. Darwin's Unfinished Symphony: How Culture Made the Human Mind. (Princeton University Press, 2017).

3 Boyd, R. A Different Kind of Animal: How Culture Transformed Our Species. (Princeton University Press, 2017).

4 Richerson, P. et al. Cultural group selection plays an essential role in explaining human cooperation: A sketch of the evidence. Behavioral and Brain Sciences 39 (2016).

5 Morin, O. How Traditions Live and Die. (Oxford University Press, 2016). 
6 Dennett, D. C. From Bacteria to Bach and Back: The Evolution of Minds. (WW Norton \& Company, 2017).

7 Youngblood, M. \& Lahti, D. A bibliometric analysis of the interdisciplinary field of cultural evolution. Palgrave Communications 4, 120 (2018).

8 Kashima, Y., Bain, P. G. \& Perfors, A. The psychology of cultural dynamics: what is it, what do we know, and what is yet to be known? Annual review of psychology $\mathbf{7 0}$, 499-529 (2019).

9 Lewens, T. Cultural Evolution: Conceptual Challenges. (Oxford University Press, 2015).

10 Sterelny, K. Cultural evolution in California and Paris. Studies in History and Philosophy of Science Part C: Studies in History and Philosophy of Biological and Biomedical Sciences 62, $42-50$ (2017).

11 Sperber, D. Explaining culture: A naturalistic approach. Cambridge, MA: Cambridge (1996).

12 Sperber, D. \& Hirschfeld, L. A. The cognitive foundations of cultural stability and diversity. Trends in cognitive sciences 8, 40-46 (2004).

13 Campbell, D. T. Blind variation and selective retentions in creative thought as in other knowledge processes. Psychological review 67, 380 (1960).

14 Campbell, D. T. in The Philosophy of Karl Popper Vol. 1 (ed P. A. Schilpp) 412-463 (Open Court, 1974).

15 Richerson, P. J. \& Boyd, R. Not By Genes Alone: How Culture Transformed Human Evolution. (University of Chicago Press, 2005).

16 Cavalli-Sforza, L. L. \& Feldman, M. W. Cultural transmission and evolution: A quantitative approach. (Princeton University Press, 1981).

17 Fay, N. et al. Applying the cultural ratchet to a social artefact: The cumulative cultural evolution of a language game. Evolution and Human Behavior 39, 300-309 (2018).

18 Muthukrishna, M. \& Henrich, J. A problem in theory. Nature Human Behaviour, 1 (2019).

19 De Block, A. \& Ramsey, G. The organism-centered approach to cultural evolution. Topoi 35, 283-290 (2016).

20 Ramsey, G. \& De Block, A. Is cultural fitness hopelessly confused? The British Journal for the Philosophy of Science 68, 305-328 (2015).

21 Blackmore, S. The Meme Machine. (Oxford University Press, 2000). 


\section{Supplement 2 - Cumulative and non-cumulative Darwinian processes}

A cumulative Darwinian process is one in which very small improvements gradually accumulate. Each small improvement spreads through the population, creating many new sites at which a further small improvement may arise by chance. This next improvement will then itself spread, creating new sites for yet another small improvement, and so on. The result is that complex, adaptive, apparently "well designed" products are gradually assembled without the need for any intelligent design. ${ }^{1-5}$

Both types of cultural selection can be cumulative but will not always be cumulative. Special further conditions are required to turn a selection process into a cumulative one. ${ }^{6}$ In particular, the transmission of traits must occur with high fidelity, so that tiny improvements (so small as to be unobvious to their inventors) that arise by chance will be inherited rather than disappearing. It is also crucial that tiny improvements reliably make a difference to fitness, so that selection can "see" them.

We hypothesize that, at an early stage in hominin evolution, cultural transmission from parents to their offspring (vertical transmission) already met the conditions for accumulation: fidelity was high owing to the sheer amount of time offspring spent with their parents, and small differences in cultural variants made a difference to a parent's reproductive success. The result was cumulative CS1. We think cumulative CS1 may even occur in some nonhuman animals, such as dolphins and chimpanzees.

By contrast, in common with many researchers who study traditions in nonhuman animals ${ }^{13}$, we hypothesize that cumulative CS2 - the faster, distinctively human type-only came along much later. On our hypothesis, it required exclusive, task-specific copying of models, achieved through explicit learning rules. Only then would tiny improvements to existing techniques have been reliably transmitted to learners, and only then would these tiny improvements have reliably made a difference to a model's ability to attract new learners.

\section{References}

1 Dawkins, R. Climbing Mount Improbable. (WW Norton \& Company, 1996).

2 Dawkins, R. The Blind Watchmaker: Why the Evidence of Evolution Reveals a Universe Without Design. (WW Norton \& Company, 1986). 
3 Dennett, D. C. Darwin's Dangerous Idea: Evolution and the Meanings of Life. (Simon and Schuster, 1995).

4 Birch, J. Inclusive fitness as a criterion for improvement. Studies in History and Philosophy of Science Part C: Studies in History and Philosophy of Biological and Biomedical Sciences 76, 101186 (2019).

5 Gardner, A. Adaptation as organism design. Biology Letters 5, 861-864 (2009).

6 Godfrey-Smith, P. Darwinian Populations and Natural Selection. (Oxford University Press, 2009). 


\section{Supplement 3 - Cognitive gadgets}

Cultural learning, the kind of learning that makes cultural evolution possible now, involves imitation, mindreading and language. Since the 1970s and 1980s, it has been assumed that each of these capabilities is a "cognitive instinct"1 designed by genetic evolution in the Pleistocene era, inherited in the DNA of each human child, and requiring minimal learning for its development. However, recent evidence from developmental psychology and social cognitive neuroscience challenges this assumption by suggesting that, like literacy, the capacities to copy new body movements, to interpret the minds of others, and to communicate using language are all constructed by learning in the context of social interaction. Rather than cognitive instincts, they are "cognitive gadgets". 2

Imitation was thought to be a cognitive instinct because influential research in the 1970s suggested that newborn human babies can copy facial expressions. ${ }^{3}$ This result was recently undermined by a large-scale study that failed to find any sign of imitation in newborns. ${ }^{4,5}$ Furthermore, evidence has emerged that the capacity to imitate develops slowly in the course of childhood ${ }^{6,7}$ and depends on social experience in which juveniles are exposed to synchronous action, optical mirrors, and imitation by other agents. ${ }^{8-10}$

Mindreading was taken to be a cognitive instinct because it depends on specialised computations and localised areas of the brain, and because people with autism find it particularly difficult to interpret thoughts and feelings. However, these inferences have been weakened by evidence that literacy, a capacity known to be culturally rather than genetically inherited, is also characterised by computational and neural specialisation, and associated with a distinctive developmental disorder (dyslexia). ${ }^{11}$ Moreover, positive evidence that mindreading is a cognitive gadget has come from research showing that the rate, sequence and extent of mindreading development depends on how much and in what ways children converse with adults about mental states. ${ }^{12-15}$

The status of language is not so clear. Many linguists and language scientists remain convinced by Chomsky's "poverty of the stimulus argument"; that language has grammatical features that could not be learned from the environments in which language develops. ${ }^{16}$ However, this view is being challenging with increasing vigour by research showing that FOXP2 is not a "language gene"17; "specific language impairment" is not, in fact, specific to language ${ }^{18}$; computers can learn complex grammatical structures without inbuilt linguistic knowledge ${ }^{19,20}$; and, in their everyday lives, children receive much more language tuition than earlier studies suggested. ${ }^{19}$ 


\section{References}

1 Pinker, S. The Language Instinct: The New Science of Language and Mind. Vol. 7529 (Penguin, 1995).

2 Heyes, C. Cognitive Gadgets: The Cultural Evolution of Thinking. (Harvard University Press, 2018).

3 Meltzoff, A. N. \& Moore, M. K. Imitation of facial and manual gestures by human neonates. Science 198, 75-78 (1977).

4 Oostenbroek, J. et al. Comprehensive longitudinal study challenges the existence of neonatal imitation in humans. Current Biology 26, 1334-1338 (2016).

5 Oostenbroek, J. et al. Re-evaluating the neonatal imitation hypothesis. Developmental science 22, e12720 (2018).

6 Jones, S. S. Imitation in infancy: The development of mimicry. Psychological Science 18, 593-599 (2007).

7 Ray, E. \& Heyes, C. Imitation in infancy: The wealth of the stimulus. Developmental science 14, 92-105 (2011).

8 Cook, R., Bird, G., Catmur, C., Press, C. \& Heyes, C. Mirror neurons: from origin to function. Behavioral and Brain Sciences 37, 177-192 (2014).

9 de Klerk, C. C., Johnson, M. H., Heyes, C. M. \& Southgate, V. Baby steps: investigating the development of perceptual-motor couplings in infancy. Developmental Science 18, 270-280 (2015).

10 de Klerk, C. C., Lamy-Yang, I. \& Southgate, V. The role of sensorimotor experience in the development of mimicry in infancy. Developmental science 22, e12771 (2019).

11 Heyes, C. M. \& Frith, C. D. The cultural evolution of mind reading. Science 344, 1243091 (2014).

12 Pyers, J. E. \& Senghas, A. Language promotes false-belief understanding: Evidence from learners of a new sign language. Psychological science 20, 805-812 (2009).

13 Shahaeian, A., Peterson, C. C., Slaughter, V. \& Wellman, H. M. Culture and the sequence of steps in theory of mind development. Developmental psychology 47, 1239 (2011).

14 Taumoepeau, M. \& Ruffman, T. Stepping stones to others' minds: Maternal talk relates to child mental state language and emotion understanding at 15, 24, and 33 months. Child development 79, 284-302 (2008).

15 Taumoepeau, M. \& Ruffman, T. Self-awareness moderates the relation between maternal mental state language about desires and children's mental state vocabulary. Journal of experimental child psychology 144, 114-129 (2016).

16 Chomsky, N. Aspects of the Theory of Syntax. Vol. 11 (MIT press, 2014).

17 Reimers-Kipping, S., Hevers, W., Pääbo, S. \& Enard, W. Humanized Foxp2 specifically affects cortico-basal ganglia circuits. Neuroscience 175, 75-84 (2011).

18 Hsu, H. J. \& Bishop, D. V. Sequence-specific procedural learning deficits in children with specific language impairment. Developmental science 17, 352-365 (2014).

19 Christiansen, M. H., Chater, N. \& Culicover, P. W. Creating language: Integrating evolution, acquisition, and processing. (MIT Press, 2016).

20 Christiansen, M. H. \& MacDonald, M. C. A usage-based approach to recursion in sentence processing. Language Learning 59, 126-161 (2009). 


\section{References (main text)}

[1] Whitehead, H. \& Rendell, L. 2014 The cultural lives of whales and dolphins, University of Chicago Press.

[2] Sargeant, B. L. \& Mann, J. 2009 Developmental evidence for foraging traditions in wild bottlenose dolphins. Animal Behaviour 78, 715-721.

[3] Allen, J., Weinrich, M., Hoppitt, W. \& Rendell, L. 2013 Network-based diffusion analysis reveals cultural transmission of lobtail feeding in humpback whales. Science 340, 485-488. [4] Krützen, M., Kreicker, S., MacLeod, C. D., Learmonth, J., Kopps, A. M., Walsham, P. \& Allen, S. J. 2014 Cultural transmission of tool use by Indo-Pacific bottlenose dolphins (Tursiops sp.) provides access to a novel foraging niche. Proceedings of the Royal Society $B$ : Biological Sciences 281, 20140374.

[5] Whiten, A., Spiteri, A., Horner, V., Bonnie, K. E., Lambeth, S. P., Schapiro, S. J. \& De Waal, F. B. 2007 Transmission of multiple traditions within and between chimpanzee groups. Current Biology 17, 1038-1043.

[6] Boesch, C. \& Tomasello, M. 1998 Chimpanzee and human cultures. Current anthropology 39, 591-614.

[7] Aplin, L. M., Farine, D. R., Morand-Ferron, J., Cockburn, A., Thornton, A. \& Sheldon, B. C. 2015 Experimentally induced innovations lead to persistent culture via conformity in wild birds. Nature 518, 538.

[8] Whiten, A., Caldwell, C. A. \& Mesoudi, A. 2016 Cultural diffusion in humans and other animals. Current opinion in Psychology 8, 15-21.

[9] Laland, K. N. \& Galef, B. G. 2009 The question of animal culture, Harvard University Press. [10] Avital, E. \& Jablonka, E. 2000 Animal Traditions: Behavioural Inheritance in Evolution. Cambridge, Cambridge University Press.

[11] Dean, L. G., Vale, G. L., Laland, K. N., Flynn, E. \& Kendal, R. L. 2014 Human cumulative culture: a comparative perspective. Biological Reviews 89, 284-301.

[12] Krützen, M., Mann, J., Heithaus, M. R., Connor, R. C., Bejder, L. \& Sherwin, W. B. 2005 Cultural transmission of tool use in bottlenose dolphins. Proceedings of the National Academy of Sciences USA 102, 8939-8943.

[13] Humle, T. 2010 How are army ants shedding new light on culture in chimpanzees? . In The Mind of the Chimpanzee: Ecological and Experimental Perspectives (eds. E. V. Lonsdorff, S. R. Ross \& T. Matsuzawa), pp. 116-127. Chicago, IL, University of Chicago Press.

[14] Sterelny, K. 2012 The Evolved Apprentice: How Evolution Made Humans Unique. Cambridge, MA, MIT Press.

[15] Henrich, J. 2016 The Secret of Our Success: How Culture is Driving Human Evolution, Domesticating Our Species, and Making Us Smarter. Princeton, NJ, Princeton University Press.

[16] Richerson, P. J. \& Boyd, R. 2005 Not By Genes Alone: How Culture Transformed Human Evolution. Chicago, IL, University of Chicago Press.

[17] Boyd, R. 2017 A Different Kind of Animal: How Culture Transformed Our Species. Princeton, NJ, Princeton University Press.

[18] Acerbi, A., Tennie, C. \& Nunn, C. L. 2011 Modeling imitation and emulation in constrained search spaces. Learning \& Behavior 39, 104-114. (DOI:10.3758/s13420-0100009-z). 
[19] van Schaik, C. P., Pradhan, G. R. \& Tennie, C. 2019 Teaching and curiosity: sequential drivers of cumulative cultural evolution in the hominin lineage. Behavioral Ecology and Sociobiology 73, 2. (DOI:10.1007/s00265-018-2610-7).

[20] Boyd, R. \& Richerson, P. J. 1985 Culture and the Evolutionary Process. Chicago, IL, University of Chicago Press.

[21] Muthukrishna, M. \& Henrich, J. 2016 Innovation in the collective brain. Philosophical Transactions of the Royal Society B: Biological Sciences 371, 20150192.

(DOI:doi:10.1098/rstb.2015.0192).

[22] Cavalli-Sforza, L. L. \& Feldman, M. W. 1981 Cultural transmission and evolution: A quantitative approach, Princeton University Press.

[23] El Mouden, C., André, J. B., Morin, O. \& Nettle, D. 2014 Cultural transmission and the evolution of human behaviour: a general approach based on the Price equation. Journal of evolutionary biology 27, 231-241.

[24] Avital, E., Jablonka, E. \& Lachmann, M. 1998 Adopting adoption. Animal Behaviour 55, 1451-1459.

[25] Birch, J. 2017 The Philosophy of Social Evolution. Oxford, Oxford University Press.

[26] González-Forero, M. \& Gardner, A. 2018 Inference of ecological and social drivers of human brain-size evolution. Nature 557, 554.

[27] Hill, K. \& Kaplan, H. 1999 Life history traits in humans: Theory and empirical studies. Annual review of anthropology 28, 397-430.

[28] Anderson, M. L. \& Finlay, B. L. 2014 Allocating structure to function: the strong links between neuroplasticity and natural selection. Frontiers in human neuroscience 7, 918.

[29] Burkart, J. M., Schubiger, M. N. \& van Schaik, C. P. 2017 The evolution of general intelligence. Behavioral and Brain Sciences 40.

[30] Heyes, C. 2018 Cognitive Gadgets: The Cultural Evolution of Thinking. Cambridge, MA, Harvard University Press.

[31] Reader, S. M., Hager, Y. \& Laland, K. N. 2011 The evolution of primate general and cultural intelligence. Philosophical Transactions of the Royal Society B: Biological Sciences 366, 1017-1027.

[32] Dennett, D. C. 2017 From Bacteria to Bach and Back: The Evolution of Minds. New York, WW Norton \& Company.

[33] Heyes, C. M., Chater, N. \& Dwyer, D. under review Sinking in: The Baldwinisation of human cognition.

[34] Heyes, C., Ray, E., Mitchell, C. \& Nokes, T. 2000 Stimulus enhancement: controls for social facilitation and local enhancement. Learning and Motivation 31, 83-98.

[35] Spence, K. W. 1937 Experimental studies of learning and the higher mental processes in infra-human primates. Psychological Bulletin 34, 806.

[36] Mineka, S., Davidson, M., Cook, M. \& Keir, R. 1984 Observational conditioning of snake fear in rhesus monkeys. Journal of abnormal psychology 93, 355.

[37] Haaker, J., Golkar, A., Selbing, I. \& Olsson, A. 2017 Assessment of social transmission of threats in humans using observational fear conditioning. nature protocols 12, 1378.

[38] Heyes, C. M. 1994 Social learning in animals: categories and mechanisms. Biological Reviews 69, 207-231.

[39] Hill, M. R., Boorman, E. D. \& Fried, I. 2016 Observational learning computations in neurons of the human anterior cingulate cortex. Nature communications 7, 12722.

[40] Bryson, J. J. 2009 Representations underlying social learning and cultural evolution. Interaction Studies 10, 77-100. 
[41] Whiten, A. \& Ham, R. 1992 On the Nature and Evolution of Imitation in the Animal Kingdom: Reappraisal of a Century of Research. In Advances in the Study of Behavior (pp. 239-283, Elsevier.

[42] Alem, S., Perry, C. J., Zhu, X., Loukola, O. J., Ingraham, T., Søvik, E. \& Chittka, L. 2016 Associative mechanisms allow for social learning and cultural transmission of string pulling in an insect. PLoS Biology 14, e1002564.

[43] Carcea, I. \& Froemke, R. C. 2019 Biological mechanisms for observational learning. Current opinion in neurobiology 54, 178-185.

[44] Happé, F., Cook, J. L. \& Bird, G. 2017 The structure of social cognition: In (ter) dependence of sociocognitive processes. Annual review of psychology 68, 243-267.

[45] Heyes, C. 2012 What's social about social learning? Journal of Comparative Psychology 126, 193.

[46] Hoppitt, W. \& Laland, K. N. 2013 Social learning: an introduction to mechanisms, methods, and models, Princeton University Press.

[47] Joiner, J., Piva, M., Turrin, C. \& Chang, S. W. 2017 Social learning through prediction error in the brain. NPJ science of learning 2, 8.

[48] Coussi-Korbel, S. \& Fragaszy, D. M. 1995 On the relation between social dynamics and social learning. Animal behaviour 50, 1441-1453.

[49] Kulahci, I. G., Ghazanfar, A. A. \& Rubenstein, D. I. 2018 Knowledgeable lemurs become more central in social networks. Current Biology 28, 1306-1310. e1302.

[50] Claidiere, N., Messer, E. J., Hoppitt, W. \& Whiten, A. 2013 Diffusion dynamics of socially learned foraging techniques in squirrel monkeys. Current Biology 23, 1251-1255.

[51] Heyes, C. 2016 Who knows? Metacognitive social learning strategies. Trends in cognitive sciences 20, 204-213.

[52] Kendal, R., Hopper, L. M., Whiten, A., Brosnan, S. F., Lambeth, S. P., Schapiro, S. J. \& Hoppitt, W. 2015 Chimpanzees copy dominant and knowledgeable individuals: implications for cultural diversity. Evolution and Human Behavior 36, 65-72.

[53] Griffiths, O. \& Le Pelley, M. E. 2019 The outcome predictability bias is evident in overt attention. Journal of Experimental Psychology: Animal Learning and Cognition 45, 290.

[54] Tankelevitch, L., Spaak, E., Rushworth, M. F. S. \& Stokes, M. G. 2020 Previously rewardassociated stimuli capture spatial attention in the absence of changes in the corresponding sensory representations as measured with MEG. The Journal of Neuroscience, JN-RM-11721119. (DOI:10.1523/JNEUROSCI.1172-19.2020).

[55] Le Pelley, M. E., Mitchell, C. J., Beesley, T., George, D. N. \& Wills, A. J. 2016 Attention and associative learning in humans: an integrative review. Psychological Bulletin 142, 1111. [56] Alon, Y., Arad, G., Pine, D. S. \& Bar-Haim, Y. 2019 Statistical learning as a predictor of attention bias modification outcome: A preliminary study among socially anxious patients. Behaviour research and therapy 112, 36-41.

[57] Heyes, C. \& Pearce, J. M. 2015 Not-so-social learning strategies. Proceedings of the Royal Society B: Biological Sciences 282, 20141709.

[58] Kendal, R. L., Boogert, N. J., Rendell, L., Laland, K. N., Webster, M. \& Jones, P. L. 2018 Social learning strategies: Bridge-building between fields. Trends in cognitive sciences 22, 651-665.

[59] Henrich, J. \& McElreath, R. 2003 The Evolution of Cultural Evolution. Evolutionary Anthropology 12, 123-135.

[60] Henrich, J. 2004 Cultural group selection, coevolutionary processes and large-scale cooperation. Journal of Economic Behavior \& Organization 53, 3-35. 
[61] Van De Waal, E., Bshary, R. \& Whiten, A. 2014 Wild vervet monkey infants acquire the food-processing variants of their mothers. Animal Behaviour 90, 41-45.

[62] Smolker, R., Richards, A., Connor, R., Mann, J. \& Berggren, P. 1997 Sponge carrying by dolphins (Delphinidae, Tursiops sp.): a foraging specialization involving tool use? Ethology 103, 454-465.

[63] Wild, S., Krützen, M., Rankin, R. W., Hoppitt, W. J., Gerber, L. \& Allen, S. J. 2019 Longterm decline in survival and reproduction of dolphins following a marine heatwave. Current Biology 29, R239-R240.

[64] Chudek, M., Heller, S., Birch, S. \& Henrich, J. 2012 Prestige-biased cultural learning: Bystander's differential attention to potential models influences children's learning. Evolution and Human Behavior 33, 46-56.

[65] Dalmaso, M., Pavan, G., Castelli, L. \& Galfano, G. 2011 Social status gates social attention in humans. Biology letters 8, 450-452.

[66] van de Waal, E., Renevey, N., Favre, C. M. \& Bshary, R. 2010 Selective attention to philopatric models causes directed social learning in wild vervet monkeys. Proceedings of the Royal Society B: Biological Sciences 277, 2105-2111.

[67] Grampp, M., Sueur, C., van de Waal, E. \& Botting, J. 2019 Social attention biases in juvenile wild vervet monkeys: implications for socialisation and social learning processes. Primates 60, 261-275.

[68] Liszkowski, U., Carpenter, M., Henning, A., Striano, T. \& Tomasello, M. 2004 Twelvemonth-olds point to share attention and interest. Developmental Science 7, 297-307. (DOI:10.1111/j.1467-7687.2004.00349.x).

[69] Stout, D., Hecht, E., Khreisheh, N., Bradley, B. \& Chaminade, T. 2015 Cognitive Demands of Lower Paleolithic Toolmaking. PLoS One 10, e0121804.

(DOI:10.1371/journal.pone.0121804).

[70] Stout, D. \& Hecht, E. E. 2017 Evolutionary neuroscience of cumulative culture. Proceedings of the National Academy of Sciences 114, 7861-7868.

[71] Putt, S. S., Wijeakumar, S., Franciscus, R. G. \& Spencer, J. P. 2017 The functional brain networks that underlie Early Stone Age tool manufacture. Nature Human Behaviour 1, 1-8. [72] Pargeter, J., Khreisheh, N. \& Stout, D. 2019 Understanding stone tool-making skill acquisition: Experimental methods and evolutionary implications. Journal of Human Evolution 133, 146-166. (DOI:https://doi.org/10.1016/i.jhevol.2019.05.010).

[73] Gowlett, J., Gamble, C. \& Dunbar, R. 2012 Human Evolution and the Archaeology of the Social Brain. Current Anthropology 53, 693-722. (DOI:10.1086/667994).

[74] Gamble, C., Gowlett, J. \& Dunbar, R. 2011 The Social Brain and the Shape of the Palaeolithic. Cambridge Archaeological Journal 21, 115-136.

(DOI:10.1017/S0959774311000072).

[75] Grove, M. 2010 The Archaeology of Group Size. In Social Brain, Distributed Mind (p. 391.

[76] Dunstone, J. \& Caldwell, C. A. 2018 Cumulative culture and explicit metacognition: a review of theories, evidence and key predictions. Palgrave Communications 4, 145.

[77] Hawkins, R. X., Goodman, N. D. \& Goldstone, R. L. 2018 The emergence of social norms and conventions. Trends in cognitive sciences.

[78] Diaconescu, A. O., Mathys, C., Weber, L. A., Daunizeau, J., Kasper, L., Lomakina, E. I., Fehr, E. \& Stephan, K. E. 2014 Inferring on the intentions of others by hierarchical Bayesian learning. PLoS computational biology 10, e1003810. 
[79] Morgan, T., Rendell, L., Ehn, M., Hoppitt, W. \& Laland, K. 2011 The evolutionary basis of human social learning. Proceedings of the Royal Society B: Biological Sciences 279, 653-662. [80] Wisdom, T. N., Song, X. \& Goldstone, R. L. 2013 Social learning strategies in networked groups. Cognitive Science 37, 1383-1425.

[81] Heyes, C. 2017 When does social learning become cultural learning? Developmental Science 20, e12350.

[82] Heyes, C. 2016 Blackboxing: social learning strategies and cultural evolution.

Philosophical Transactions of the Royal Society B: Biological Sciences 371, 20150369.

[83] French, J. C. 2016 Demography and the Palaeolithic Archaeological Record. Journal of Archaeological Method and Theory 23, 150-199. (DOI:10.1007/s10816-014-9237-4).

[84] Heyes, C. 2016 Homo imitans? Seven reasons why imitation couldn't possibly be associative. . Philosophical Transactions of the Royal Society B: Biological Sciences 371, 20150069.

[85] Binford, L. 2001 Constructing Frames of Reference: An Analytical Method for Archaeological Theory Building Using Ethnographic and Environmental Data Sets. Berkeley, $\mathrm{CA}$, University of California Press.

[86] De Waal, F. B. \& Bonnie, K. E. 2009 In tune with others: the social side of primate culture. The question of animal culture, 19-39.

[87] Johnson, M. H., Senju, A. \& Tomalski, P. 2015 The two-process theory of face processing: modifications based on two decades of data from infants and adults.

Neuroscience \& Biobehavioral Reviews 50, 169-179.

[88] Vouloumanos, A. \& Werker, J. F. 2007 Listening to language at birth: Evidence for a bias for speech in neonates. Developmental science 10, 159-164.

[89] Perea-García, J. O., Kret, M. E., Monteiro, A. \& Hobaiter, C. 2019 Scleral pigmentation leads to conspicuous, not cryptic, eye morphology in chimpanzees. Proceedings of the National Academy of Sciences, 201911410.

[90] Tennie, C., Premo, L. S., Braun, D. R. \& McPherron, S. P. 2017 Early stone tools and cultural transmission: Resetting the null hypothesis. Current anthropology: A world journal of the sciences of man, 652-672.

[91] Stout, D. \& Khreisheh, N. 2015 Skill learning and human brain evolution: An experimental approach. Cambridge Archaeological Journal 25, 867-875.

[92] Biro, D., Inoue-Nakamura, N., Tonooka, R., Yamakoshi, G., Sousa, C. \& Matsuzawa, T. 2003 Cultural innovation and transmission of tool use in wild chimpanzees: evidence from field experiments. Anim Cogn 6, 213-223. (DOI:10.1007/s10071-003-0183-x).

[93] Biro, D., Sousa, C. \& Matsuzawa, T. 2006 Ontogeny and Cultural Propagation of Tool Use by Wild Chimpanzees at Bossou, Guinea: Case Studies in Nut Cracking and Leaf Folding. In Cognitive Development in Chimpanzees (eds. T. Matsuzawa, M. Tomonaga \& M. Tanaka), pp. 476-508. Tokyo, Springer Tokyo. 